\title{
Splenic Artery Aneurysm. A Rare Entity Case Report
}

\author{
Md. Danny Fernando Silva Cevallos ${ }^{1 *}$, Md. Mirella Barrera ${ }^{2}$, \\ Md. Fernando Andrés Silva Michalón ${ }^{3}$, Md. Marcos Leonardo Matute \\ Rivera $^{4}$, Md. Ernesto Kang Moreira ${ }^{5}$ and Manuel Velimir Flores Delgado ${ }^{6}$ \\ ${ }^{1}$ Internist/Head of Emergencies and Hospitalization, Guayaquil Clinic, City \\ Guayaquil, Ecuador \\ ${ }^{2}$ Medical Service of Internal Medicine, Clinic Guayaquil/UESS, Ecuador \\ ${ }^{3}$ Independent Medical Researcher/Universidad Catolica, Ecuador \\ ${ }^{4}$ General Surgeon, Clínica Guayaquil/UESS, Ecuador \\ ${ }^{5}$ Medical Internal Medicine Service, Clínica Guayaquil/UESS, Ecuador \\ ${ }^{6}$ Medical Internal Medicine Service, Guayaquil Clinic/UESS, Ecuador \\ *Corresponding Author: Md. Danny Fernando Silva Cevallos, Internist/Head of \\ Emergencies and Hospitalization, Guayaquil Clinic, City Guayaquil, Ecuador.
}

\author{
Received: October 29, 2021 \\ Published: November 23, 2021 \\ (C) All rights are reserved by Md. Danny \\ Fernando Silva Cevallos., et al.
}

\begin{abstract}
Splenic artery aneurysms are rare entities, their prevalence is estimated between 0.9 to $9.7 \%$. They are asymptomatic, and with the help of imaging diagnosis, incidental findings have increased. Once diagnosed, treatment is proposed, which can be surgical, such as splenectomy and aneurysm resection when they have a diameter greater than 2cc, endovascular by embolization or placement of stent.

We present the case of a 36-year-old patient with a history of insulin-dependent type 1 Diabetes Mellitus, who reported sudden, burning, cramping abdominal pain in the left upper quadrant, accompanied by melena.

Keywords: Aneurysm; Splenic; Endovascular; Splenectomy

\section{Introduction}

The aneurysm of the splenic artery is defined as the dilation of this greater than $1 \mathrm{~cm}$ are presented up to $60 \%$ being the third cause of all abdominal aneurysms, after the aorta and iliac arteries. Vascular diseases of the spleen are uncommon, present with nonspecific clinical manifestations and with significant morbidity and mortality, the affectation mostly occurs in women four times more than men, in patients who are asymptomatic and is evidenced by tomography with a diameter of $>2 \mathrm{~cm}$ or continues to increase in

size should be treated by means of transcatheste embolization or failing that, high-risk patients and in those surgically difficult to approach will require splenectomy [1].

The pathogenesis of this pathology is not clear, but its risk factors are included such as atherosclerosis 32\%, trauma $22 \%$, fibrodysplasia of the splenic artery $24 \%$, Arterial hypertension, infectious processes, pregnancies in 10\%, alterations of connective tissue (Marfán syndrome, Ehlen Danlos syndrome, Kawasaki syndrome) [2,3].
\end{abstract}




\section{Clinical Case Description}

A 36-year-old male patient with a personal pathological history of Diabetes mellitus tipo 1 treated with insulin 70/30 (20IU AM - 10 IU PM). vital signs: TA 107/66 Hg mm, FC76, FC 14. Hgt $151 \mathrm{~mm} / \mathrm{dl}$. With clinical picture of 15 days of evolution characterized by abdominal pain type colic of moderate intensity, located at the level of epigastrium that radiates to the left hypochondrium and posterior thoracic region, accompanied by nausea that progresses to vomit in number of $4 / 24 \mathrm{~h}$ of food content. In addition to abundant manes on several occasions, I present lipothymia, to the physicalxamen marked paleness, lethargic, mucousmarries, soft abdomen, depressible pain of moderate to great intensity in left hypochondrium that radiates towards hypogastrium.

By presumptive diagnosis of the patient is made inter consultation to the hemodynamics service who determines to perform arteriography where they report splenic artery aneurysm of difficult access so it requests assessment to the vascular surgery service, valued by said service determining therapeutic behavior performing exploratory laparotomy procedure with findings splenic artery aneurysm of approximately $10 \mathrm{c} \mathrm{m}$ approximately, between arteries trunk celiac and superior mesenteric, pulsatile and with flow in ultrasound by localization it is not possible to perform excéres is of the same.

\begin{tabular}{|l|c|}
\hline \multicolumn{1}{|c|}{ Major clinical features } & $\begin{array}{c}\text { Approximate \% } \\
\text { of patients }\end{array}$ \\
\hline Mucocutaneous triad & 89 \\
\hline Abnormal skin pigmentation & 88 \\
\hline Nail dystrophy & 78 \\
\hline Leukoplakia & 85.5 \\
\hline Bone marrow failure & 30.5 \\
\hline Other recognized somatic features (Minor) & 25.4 \\
\hline Epiphora & \\
\hline $\begin{array}{l}\text { Learning difficulties/developmental delay/ } \\
\text { mental retardation }\end{array}$ & 20.3 \\
\hline Pulmonary disease & 19.5 \\
\hline Short stature & 16.9 \\
\hline Extensive dental caries/loss & 16.9 \\
\hline Esophageal stricture & 16.1 \\
\hline $\begin{array}{l}\text { Premature hair loss/greying/sparse eye- } \\
\text { lashes }\end{array}$ & \\
\hline
\end{tabular}

22

\begin{tabular}{|l|c|}
\hline Hyperhiderosis & 15.3 \\
\hline Malignancy & 9.8 \\
\hline Intrauterine growth retardation & 7.6 \\
\hline Liver disease/peptic ulceration/enteropathy & 7.3 \\
\hline Ataxia/cerebellar hypoplasia & 6.8 \\
\hline Hypogonadism/undescended testes & 5.9 \\
\hline Microcephaly & 5.9 \\
\hline Urethral stricture/phimosis & 5.1 \\
\hline Osteoporosis/aseptic necrosis/scoliosis & 5.1 \\
\hline Deafness & 0.8 \\
\hline
\end{tabular}

Table 1: Multisystem clinical features of dyskeratosis congenital $[3,5]$.

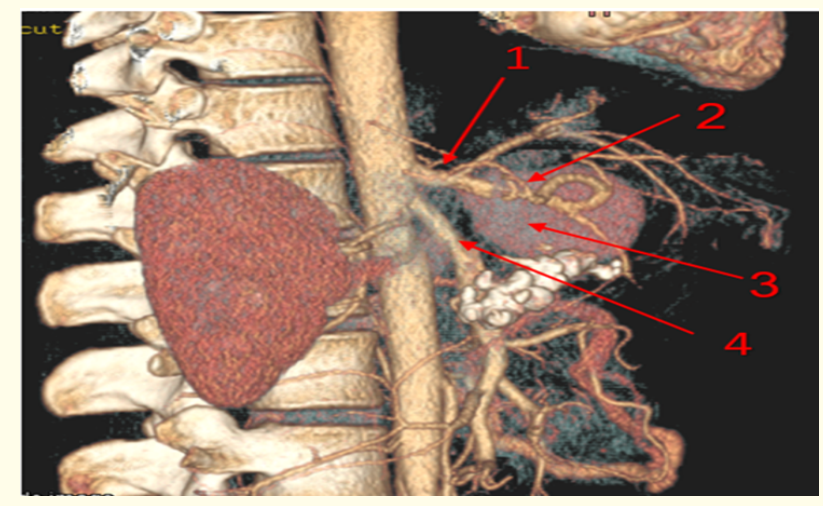

Figure 1

1. Celiac Trunk; 2. Splenic artery; 3. Aneurysm; 4. Upper Mesenteric.

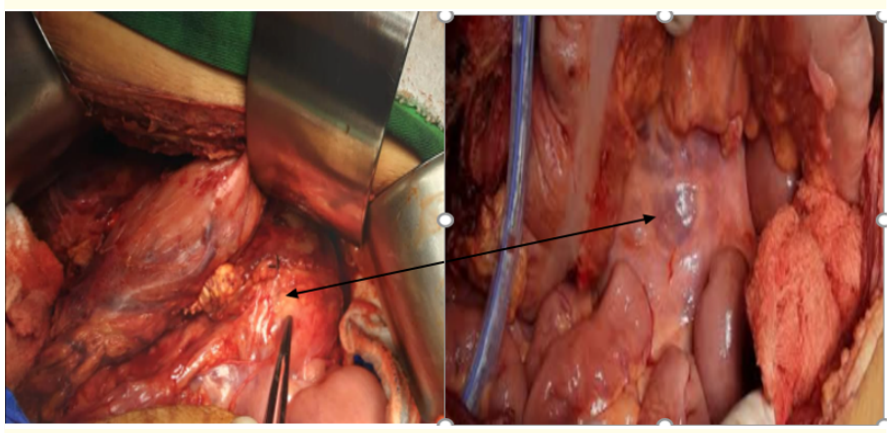

Figure 2: Evidence of splenic aneurysm. 


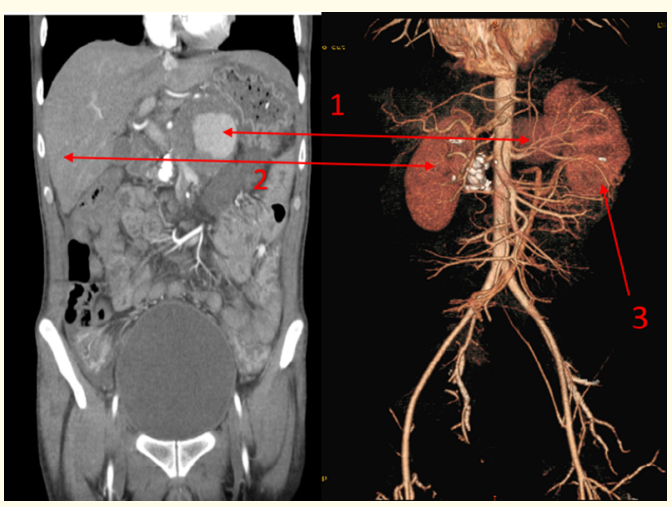

Figure 3

1. Aneurysm with splenic mural thrombus, pancreatic calcifications; 2. Liver; 3. Left kidney.

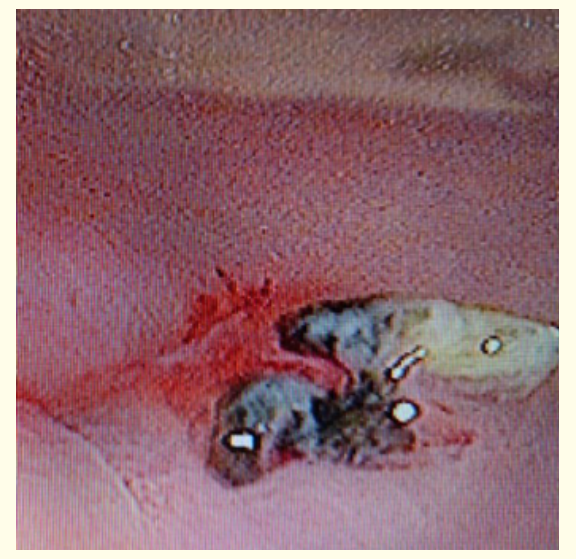

Figure 4: Upper digestive endoscopy. clots are evident in the gastric anthro mucosa.

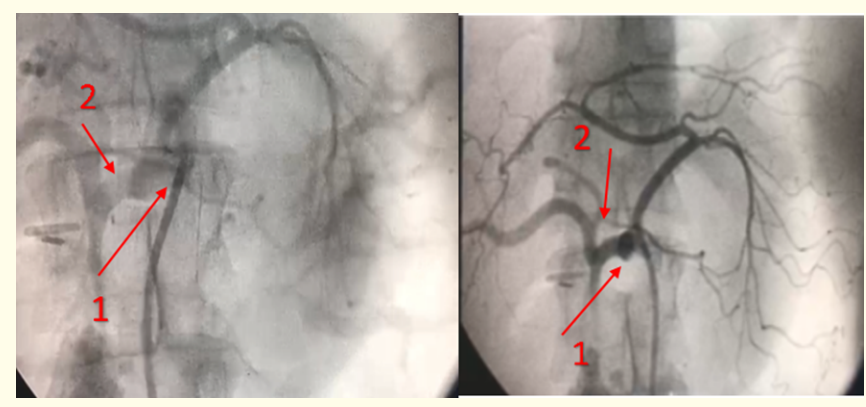

Figure 5: Arteriography.

1- Aneurysm area; 2. Celieaco trunk.

\section{Discussion}

Splenic artery aneurysms are regularly predominant in women with a 4: 1 ratio for men, it is detected mostly in multiparous up to $80 \%$, being asymptomatic, they are regularly findings detected through imaging studies. When they present symptoms they are nonspecific as pain in the hypochondrium or left flank. The main complication AAE is rupture and its risk varies between 2 to $10 \%$, which often occurs in pregnancy with a rate ranging from 20 to $50 \%$. Among other complications may occur embolism, thrombosis fistulas of neighboring structures, such as pancreas or digestive tract causing hemorrhages or (hemosuccus pancreaticus). Mortality after rupture varies between $25 \%$ and $70 \%$ several treatments exist in relation to splenic aneurysms, such as embolization, aneurysmectomy or splenectomy.

Pseudoaneurysm of the splenic artery can also be found with less prevalence of predominance of the male sex 2:1 and are related to a history of pancreatitis or trauma, they are usually chronic processes including pancreatic pseudocysts, these are of poor prognosis with a high risk of digestive bleeding around $50 \%$ and mortality of 50\% [3]. The existence of an arteriovenous fistula can cause portal hypertension, ascites, splenomegaly or esophageal varices as well as is associated with diarrhea in relation to increased pressure in the mesenteric vein, by this same mechanism can develop heart failure.

Aneurysms larger than $2 \mathrm{~cm}$ rarely rupture, but giant aneurysms their risk of rupture may be to the peritoneum causing hemoperitoneum and hemorrhagic shock $2-10 \%$, or rupture in two times or double rupture between $20-30 \%$ of cases, consists of a self-limited hemorrhage that is located in the transcavity of the omentums, then in a second time it gives rise to a massive hemorrhage when communicating with Wilson's hiatus and with the free peritoneum. As well as the rupture in hollow viscera such as stomach which causes hematemesis and manes or the colon causing digestive bleeding.

Patients treated surgically on an elective basis may have an intra- or postoperative mortality of around 0.5 to $5 \%$. The highest incidence of this disease occurs in young pregnant women, with more than $95 \%$ of the aneurysms detected rupture with what leads to a maternal mortality of more than $70 \%$ and a fetal mortality equally greater than $75 \%$, another group of patients of high mortality are those of liver transplants so the primary recommendation is the thorough review of the splenic artery before transplantation. 
Its diagnosis is given by the images that are an important role. An X-ray of the abdomen of the abdomen may appear as a rounded calcification in the area of the splenic artery in up to $70 \%$ of cases [4].

Ultrasound is frequently used in the detection of aortic aneurysms with low frequency $4 \mathrm{MHz}$ translators with Doppler and grayscale analysis, which allows estimating the size of the aneurysm.

Tomography or CT angiography performs the standard method for confirming the diagnosis, whether it is aneurysm or pseudoaneurysm. Three-phase or multi-phase CT scan in different phases can accurately and clearly show the location, shape, extent, measurement, aneurysmal wall, affected artery and relationship with adjacent vessels or structures. Angioresonance is an alternative for people who have tomographic contraindication such as allergy to ioda substances [5].

There is still no consensus on what the treatment of splenic aneurysm is. several authors postulate or recommend the conservative management of small and asymptomatic aneurysms on elderly people assessing their growth with frequent or periodic controls.

But other authors conclude and agree on the early treatment in patients with symptoms, large ruptured aneurysms $>2 \mathrm{~cm}$, pseudoaneurysm and in pregnant women, several therapeutic options are included as: endovascular treatment with embolization or stenting, splene aneurysmectomy, aneurysmal ligation, vascular resection with primary anastomosis or gralt interposition, this procedure preserves the spleen but involves more surgical time and the operation dor must have good training in vascular reconstruction.

Emergency splenectomy in a procedure that involves high morbidity and mortality that is around $22 \%$, this therapy should be reserved for unrecontic aneurysmal rupture.

Endovascular embolization is a good treatment option with favorable success rates reaching up to 80 to $90 \%$, two main techniques are mentioned to treat a saccular aneurysm: packing or filling of the aneurysm and isolation by arterial embolization, first the distal part and then proximal of the aneurysm in the form of a sandwich, or by occlusion of the carrier vessel [6].
Splenic artery aneurysms are rare, with a low incidence, their diagnosis is usually incidental in imaging studies, they are often asymptomatic. Its diagnosis and treatment is a challenge for the multidisciplinary group. It should be indicated that endovascular treatment has a favorable success, as well as those of endoscopic type. Surgical must take into account the high percentage of mortality that this entails. It does not hurt to always establish the best criteria and method of approach, unless all efforts are exhausted, conservative treatment will be indicated.

In this case it was evidenced from the natural history of the disease con antecedent of hypertension arterial, with clinical picture of abdominal pain in left hypochondrium and with ultrasound evidence abdominal the process of research Ratified with tomography it is specified that the patient have an artery aneurysm Splenic than approximately 7,2 x 6.6.Cm axial and coronal $6.4 \times 6.5 \mathrm{~cm}$ ) aneurysm with mural thrombus of splenic artery. In addition is evidenced than the patient Presented Complications how chronic pancreatitis with organ pancreatic calcificado fully visible in the images, Present haemorrhage Digestive loud severe followed by manes, because of the mouth towards viscera Hollow as it was in this case that zone he was visualized hemorrhagic in the pyloric anthro, verified in endoscopy, was not performed sclerosis of this area since could compromise the life of the patient.

In relation to our case, conservative treatment was estimated by a difficult approach during attempt to embolization and/or possibility of placement of stent which were failed In addition by the proximity of the splenic artery aneurysm giant to the celiac trunk and mesenteric artery superior, with high riesmortality go for exercises the ligature of this me Also a surgical procedure Was deceased por the causes mentioned above frame a great a fortuitous challenge for the multidisciplinary team. Discharged in better conditions so beta-blockers, antihypertensive were indicated and improve lifestyle.

It is concluded that this patient presented natural history related to the pathology, that the methods of diagnosis by images defined the dimension of the aneurysm, all the intervention protocols were complied with being failed.

\section{Conclusion}

This work focuses on splenic artery aneurysm, which is a rare clinical condition; however, it can lead to life-threatening bleeding.

Citation: Md. Danny Fernando Silva Cevallos., et al. "Splenic Artery Aneurysm. A Rare Entity Case Report". Acta Scientific Gastrointestinal Disorders 4.12 (2021): 21-25. 
Currently, endovascular interventions are the primary treatment option for splenic artery aneurysm. However, they do not apply to all patients and it may be necessary to choose the right surgery, the main goal should be minimally invasive procedures that require a good laparoscopic experience.

\section{Bibliography}

1. Batista MEM., et al. "Splenic artery aneurysm: Incidental finding".

2. Hernandez Moreno LC., et al. "Giant splenic artery aneurysm. Report of a case". Revista de Cirugia 72.4 (2021).

3. Cabral D., et al. "Splenic artery aneurysm. About a case". Cirugía Paraguaya 44.3(2020):33-34.

4. Ruiz de la Hermosa A., et al. "Splenic aneurysm associated with a hilar arteriovenous fistula". Hepatology and Gastroenterology 34.6 (2011): 393-397.

5. Usta MA., et al. "Splenic artery aneurysm, uncommon cause of abdominal pain. Presentation of a case".

6. Vanetta C., et al. "Endovascular treatment of incidental and emergency splenic aneurysm". Medicina (B Aires) 81.1 (2021): 96-98.

Volume 4 Issue 12 December 2021

(C) All rights are reserved by Md. Danny Fernando Silva Cevallos., et al. 